\title{
Pyogenic Liver Abscess With Complicating Intestinal Tuberculosis
}

\author{
Nae-Yun Heo a, d, Seung Ha Park ${ }^{\mathrm{a}}$, Jongha Park ${ }^{\mathrm{a}}$, Tae Oh Kimª, \\ Young Soo Moon ${ }^{\mathrm{a}}$, Jung-Hee Yoon ${ }^{\mathrm{b}}$, Chae Hun Lee ${ }^{\mathrm{c}}$
}

\begin{abstract}
Colonic diseases can cause pyogenic liver abscess in the case of diverticulitis, inflammatory bowel disease, colon cancer or after iatrogenic colon polypectomy. However, there is no report pyogenic liver abscess related to intestinal tuberculosis until now. We report the case of a 60 -year-old woman with a pyogenic liver abscess, who was infected with Klebsiella pneumoniae and had complicating intestinal tuberculosis in the colon, which was probably the source of the liver abscess. This case suggests that intestinal tuberculosis should be considered as one of the possible causes for cryptogenic pyogenic liver abscess.
\end{abstract}

Keywords: Liver abscess; Intestinal tuberculosis

\section{Introduction}

Currently, the most frequent cause of pyogenic liver abscess is biliary disease. And the inflammation or mucosal defects of the colon are second common cause of pyogenic liver abscess in the case of diverticulitis, inflammatory bowel disease, colon cancer or after iatrogenic colon polypectomy [1-5]. Intestinal tuberculosis develops the intestinal mucosal defect, but there has been no report of pyogenic liver abscess associated with it. We present the case of a 60 -year-

\footnotetext{
Manuscript accepted for publication August 13, 2012

${ }^{\text {a } D e p a r t m e n t ~ o f ~ I n t e r n a l ~ M e d i c i n e, ~ H a e u n d a e ~ P a i k ~ H o s p i t a l, ~ I n j e ~}$ University College of Medicine, Busan, Korea

${ }^{\mathrm{b}}$ Department of Radiology, Haeundae Paik Hospital, Inje University College of Medicine, Busan, Korea

${ }^{c}$ Department of Family Medicine, Busan Paik Hospital, Inje University College of Medicine, Busan, Korea

${ }^{\mathrm{d}}$ Corresponding author: Nae-Yun Heo, Department of Internal Medicine, Haeundae Paik Hospital, Inje University College of Medicine, Busan, 1435, Jwa-dong, Haeundae-gu, Busan, 612-030, Korea. Email: nyheo@hanmail.net
}

doi: http://dx.doi.org/10.4021/jmc844w old woman with a pyogenic liver abscess, who was infected with Klebsiella pneumoniae and had complicating intestinal tuberculosis in the colon, which was probably the source of the liver abscess.

\section{Case Report}

A previously healthy 60 -year-old female was admitted to the emergency room complaining of abdominal pain. Her abdominal pain had developed 3 days previously and had a diffuse, intermittent cramping pattern; she was sometimes awakened at night because of the pain. She had no specific past medical history except cholelithiasis, which had been treated by a cholecystectomy at the age of 28 years. She denied a history of smoking and alcohol intake. Upon physical examination, she appeared acutely ill and presented with diffuse abdominal tenderness with muscle guarding and mild rebound tenderness. Her body temperature was $36.9^{\circ} \mathrm{C}$. Leukocytosis was shown $11,250 \mathrm{cells} / \mathrm{mm}^{3}$ (neutrophil, $89.6 \%$ ). The inflammatory markers were elevated such that C-reactive protein was $>40 \mathrm{mg} / \mathrm{dL}$ and procalcitonin was 58.05 $\mu \mathrm{g} / \mathrm{L}$. An initial abdominal computed tomography (CT) scan showed a $5.5 \times 3.5 \mathrm{~cm}$, oval-shaped, heterogeneously low attenuated lesion in the left lateral segment of the liver (Fig.1).

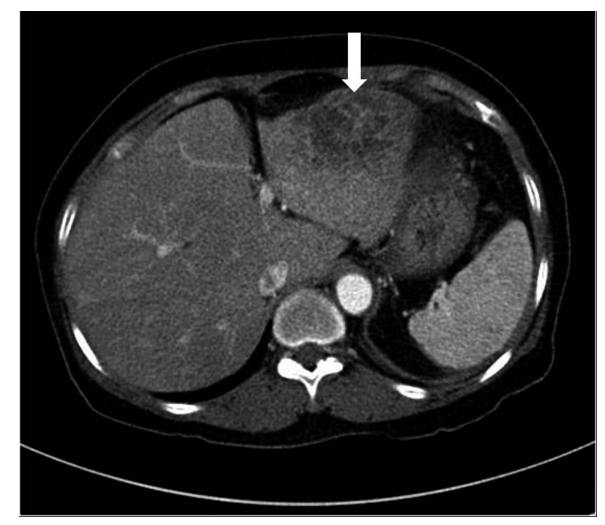

Figure 1. The initial CT scan shows a $5.5 \times 3.5 \mathrm{~cm}$, oval shaped, heterogeneously low attenuated lesion in the left lateral segment of the liver (white arrow). 


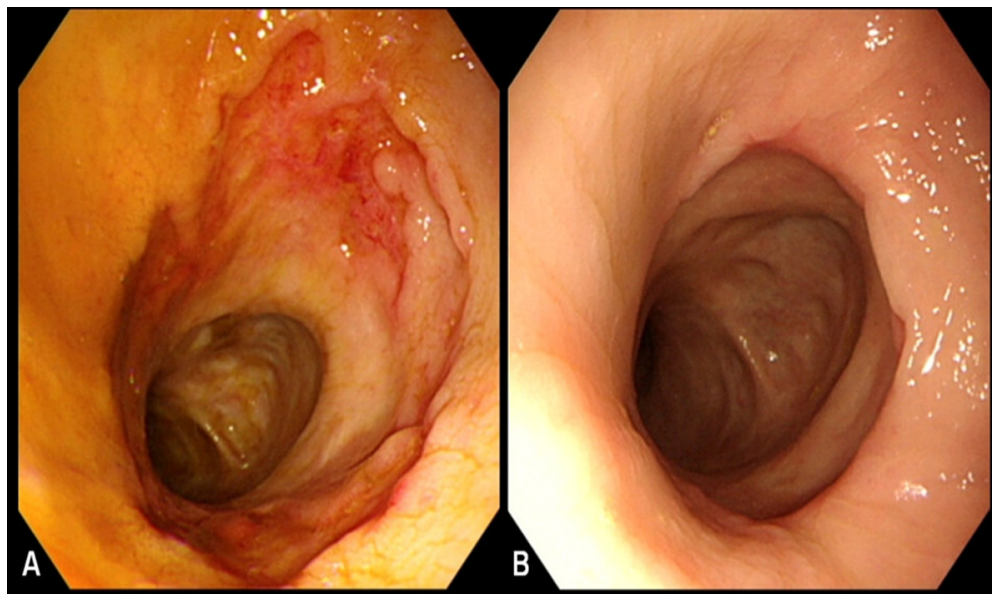

Figure 2. The initial colonoscopic examination shows a transverse ulcer with an irregular margin in the mid-ascending colon. Multiple mucosal scars were shown proximal to the ulcer (A). After the completion of 4 weeks of anti-tuberculosis medications, the transverse ulcer in the mid-ascending colon was healed (B).

We diagnosed the disease as liver abscess and performed blood cultures and administered the empirical antibiotics cefotaxime and metronidazole intravenously. The results of the blood and abscess cultures were then reported to be positive for Klebsiella pneumonia. The serum amebic antibody test was negative.

Following the injection of antibiotics, the patient complained of anorexia and diarrhea and had coffee-colored, tarry stools. We performed an endoscopic exam of the upper and lower gastrointestinal tracts on hospital day 6 with the intention to identify antibiotic-associated colitis or the focus of the gastrointestinal bleeding. Chronic superficial gastritis was shown by the upper gastrointestinal endoscopy. The colonoscopic examination showed a transverse ulcer with an irregular margin in the mid-ascending colon, a patulous ileocecal value, and multiple mucosal scar changes on the cecum and proximal ascending colon (Fig. 2A). The pathology of the ulcer in the mid-ascending colon showed acute and chronic colitis with granuloma formation and multiple erosions; however, the AFB stain was negative and $\mathrm{Myco}$ bacterium tuberculosis did not grow on tissue culture. We assessed the condition as intestinal tuberculosis on the basis of the endoscopic and pathologic findings and then prescribed anti-tuberculosis quadri-therapy with isoniazid, rifampicin, pyrazinamide and ethambutol. After beginning the anti-tuberculosis medications, the patient improved and her diarrhea disappeared.

After completing a 2-week course of intravenous antibiotics, the patient was discharged with oral cefixime and anti-tuberculosis medications. The ultrasonographic findings 2 weeks after her discharge showed that the liver abscess was organized and had shrunk. The colonoscopic examination 3 weeks after discharge showed that the transverse ulcer in the mid-ascending colon had healed (Fig. 2B).

\section{Discussion}

After the introduction of antibiotics, pylephlebitis complications during appendicitis became a rare cause of pyogenic liver disease. Currently, infections of the biliary tract, such as cholangitis and cholecystitis, are the most common identified source of pyogenic liver abscess [1]. However, 5-15\% of pyogenic liver abscess cases are caused by an infectious pathogen in the portal vein and are associated with diverticulitis [2], inflammatory bowel disease [3], gastrointestinal malignancy, and perforated peptic ulcer. Moreover, Jeong et al reported that 9 out of 37 patients (24\%) with cryptogenic pyogenic liver abscess who underwent colonoscopy had a colonic cause, 6 of which were proven to be colon cancer [4]. Moreover, the case of pyogenic liver abscess following colon polypectomy has been reported [5]. Therefore, these results suggest that a portion of the cases of pyogenic liver abscess is due to colonic mucosal defects, and colonic investigation should be considered in the case of cryptogenic liver abscess. According to our knowledge, despite the relationship between pyogenic liver abscess and colonic mucosal defects, there has been no report on pyogenic liver abscess with complicating intestinal tuberculosis, which usually causes chronic inflammatory ulcerative lesions on the colon mucosa.

Although the patient in this case had a cholecystectomy due to cholethiasis, there was no evidence of remaining intra- or extra-hepatic bile duct stones in the abdominal CT scan. In addition, there were no other intestinal mucosal defects except for a transverse ulcer in the ascending colon. The identified pathogen, Klebsiella pneumoniae, is known as a colonizer in $3-35 \%$ of the colons of healthy humans [6]. Therefore, this pyogenic liver abscess was probably associated with the mucosal lesion of intestinal tuberculosis. Polat 
et al reported a young male patient with Down's syndrome who presented with tuberculous liver abscess secondary to obstructive and multiple perforated ileal tuberculosis [7]. However, our report is the first worldwide of pyogenic (not tuberculous) liver abscess with complicating intestinal tuberculosis to our knowledge.

We suggest that there are two hypotheses explaining the formation of this case of liver abscess. One hypothesis is the hematogenous bacterial spreading with mucosal disruption and underlying blood vessel exposure due to the ulcerative lesion from the intestinal tuberculosis. The second hypothesis is the direct extension from the bowel lumen to the liver through microperforation. The detection of a single isolate (not mixed) supports the hematogenous spread theory [8], but the location of the abscess abutting the capsule suggests the possibility of direct spreading of the bacteria through the intraperitoneal cavity.

This case suggests that intestinal tuberculosis should be considered as one of the possible causes for cryptogenic pyogenic liver abscess, especially with abdominal symptoms, such as abdominal pain, diarrhea, melena; thus, adequate colonic investigation may help to determine the cause of pyogenic liver abscess.

\section{Conflict of Interest}

No conflict of interest.

\section{Grant Support}

No funding.

\section{References}

1. Sifri CD, Madoff LC. Infection of the liver and biliary system. In: Mandell GL, Bennett JE, Dolin R, editors. Mandell, Douglas, and Bennett's Principles and practice of infectious diseases. 7th ed. Philadelphia: Churchill Livingstone/Elsevier; 2010. p.1035-6.

2. Knowles R, Rinaldo JA. Pyogenic liver abscess probably secondary to sigmoid diverticulitis: report of two cases. Gastroenterology. 1960;38:262-266.

3. Margalit M, Elinav H, Ilan Y, Shalit M. Liver abscess in inflammatory bowel disease: report of two cases and review of the literature. J Gastroenterol Hepatol. 2004;19(12):1338-1342.

4. Jeong SW, Jang JY, Lee TH, Kim HG, Hong SW, Park $\mathrm{SH}$, Kim SG, et al. Cryptogenic pyogenic liver abscess as the herald of colon cancer. J Gastroenterol Hepatol. 2012;27(2):248-255.

5. Farmer AD, Browett K, Rusius V, Bhalerao S, Anderson MR. Pyogenic liver abscess as a complication of sigmoid polypectomy. Endoscopy. 2007;39 Suppl 1:E261.

6. Russo TA, Johnson JR. Diseases caused by gram-negative enteric bacilli. In: Longo DL, Harrison TR, editors. Harrison's principles of internal medicine. 18th ed. New York: McGraw-Hill; 2012. P.1253.

7. Polat KY, Aydinli B, Yilmaz O, Aslan S, Gursan N, Ozturk G, Onbas O. Intestinal tuberculosis and secondary liver abscess. Mt Sinai J Med. 2006;73(6):887-890.

8. Baron MJ, Kasper DL. Intraabdominal infections and abscesses. In: Longo DL, Harrison TR, editors. Harrison's principles of internal medicine. 18th ed. New York: McGraw-Hill; 2012. p.1081. 